\title{
A New Generating Function of ( $q$-) Bernstein-Type Polynomials and Their Interpolation Function
}

\author{
Yilmaz Simsek $^{1}$ and Mehmet Acikgoz ${ }^{2}$ \\ ${ }^{1}$ Department of Mathematics, Faculty of Arts and Science, University of Akdeniz, 07058 Antalya, Turkey \\ 2 Department of Mathematics, Faculty of Arts and Science, University of Gaziantep, \\ 27310 Gaziantep, Turkey
}

Correspondence should be addressed to Mehmet Acikgoz, acikgoz@gantep.edu.tr

Received 19 January 2010; Accepted 1 March 2010

Academic Editor: Lance Littlejohn

Copyright ( 2010 Y. Simsek and M. Acikgoz. This is an open access article distributed under the Creative Commons Attribution License, which permits unrestricted use, distribution, and reproduction in any medium, provided the original work is properly cited.

\begin{abstract}
The main object of this paper is to construct a new generating function of the $(q-)$ Bernsteintype polynomials. We establish elementary properties of this function. By using this generating function, we derive recurrence relation and derivative of the $(q-)$ Bernstein-type polynomials. We also give relations between the $(q-)$ Bernstein-type polynomials, Hermite polynomials, Bernoulli polynomials of higher order, and the second-kind Stirling numbers. By applying Mellin transformation to this generating function, we define interpolation of the $(q-)$ Bernstein-type polynomials. Moreover, we give some applications and questions on approximations of $(q-)$ Bernstein-type polynomials, moments of some distributions in Statistics.
\end{abstract}

\section{Introduction}

In [1], Bernstein introduced the Bernstein polynomials. Since that time, many authors have studied these polynomials and other related subjects (cf, [1-25]), and see also the references cited in each of these earlier works. The Bernstein polynomials can also be defined in many different ways. Thus, recently, many applications of these polynomials have been looked for by many authors. These polynomials have been used not only for approximations of functions in various areas in Mathematics, but also for the other fields such as smoothing in statistics, numerical analysis and constructing Bezier curve which have many interesting applications in computer graphics (cf, $[1,5,7,13-20,25]$ and see also the references cited in each of these earlier works).

The $(q-)$ Bernstein polynomials have been investigated and studied by many authors without generating function. So far, we have not found any generating function of $(q-)$ Bernstein polynomials in the literature. Therefore, we will consider the following question 
The aim of this paper is to give answer this question and to construct generating function of the $\left(q^{-}\right)$Bernstein-type polynomials which is given in Section 3. By using this generating function, we not only give recurrence relation and derivative of the $(q-)$ Bernsteintype polynomials, but also find relations between higher-order Bernoulli polynomials, the Stirling numbers of the second-kind, and the Hermite polynomials. In Section 5, by applying Mellin transformation to the generating function of the $(q-)$ Bernstein-type polynomials, we define interpolation function, which interpolates the $(q-)$ Bernstein-type polynomials at negative integers.

\section{Preliminary Results Related to the Classical Bernstein, Higher-Order Bernoulli, and Hermit Polynomials as well as the Stirling Numbers of the Second-Kind}

The Bernstein polynomials play a crucial role in approximation theory and the other branches of Mathematics and Physics. Thus in this section we give definition and some properties of these polynomials.

Let $f$ be a function on $[0,1]$. The classical Bernstein polynomials of degree $n$ are defined by

$$
\mathbb{B}_{n} f(x)=\sum_{j=0}^{n} f\left(\frac{j}{n}\right) B_{j, n}(x), \quad 0 \leq x \leq 1,
$$

where $\mathbb{B}_{n} f$ is called the Bernstein operator and

$$
B_{j, n}(x)=\left(\begin{array}{l}
n \\
j
\end{array}\right) x^{j}(1-x)^{n-j},
$$

$j=0,1, \ldots, n$ are called the Bernstein basis polynomials (or the Bernstein polynomials of degree $n$ ). There are $n+1 n$th degree Bernstein polynomials. For mathematical convenience, we set $B_{j, n}(x)=0$ if $j<0$ or $j>n$ (cf, $[1,5,7,9,14,18-20]$ ).

If $f:[0,1] \rightarrow \mathbb{C}$ is a continuous function, then the sequence of Bernstein polynomials $\mathbb{B}_{n} f(x)$ converges uniformly to $f$ on $[0,1]$ (cf, [10]).

A recursive definition of the $k$ th $n$th Bernstein polynomials can be written as

$$
B_{k, n}(x)=(1-x) B_{k, n-1}(x)+x B_{k-1, n-1}(x)
$$

For proof of the above relation see [9].

For $0 \leq k \leq n$, derivatives of the $n$th degree Bernstein polynomials are polynomials of degree $n-1$ :

$$
\frac{d}{d t} B_{k, n}(t)=n\left(B_{k-1, n-1}(t)-B_{k, n-1}(t)\right)
$$

(cf, $[1,5,7,9,14,18,19])$. On the other hand, in Section 3, using our new generating function, we give the other proof of (2.4). 
Observe that the Bernstein polynomial of degree $n, \mathbb{B}_{n} f$, uses only the sampled values of $f$ at $t_{n j}=j / n, j=0,1, \ldots, n$. For $j=0,1, \ldots, n$,

$$
\beta_{j, n}(x) \equiv(n+1) B_{j, n}(x), \quad 0 \leq x \leq 1,
$$

is the density function of beta distribution $\operatorname{bet} a(j+1, n+1-j)$.

Let $y_{n}(x)$ be a binomial $b(n, x)$ random variable. Then

$$
\begin{gathered}
E\left\{y_{n}(x)\right\}=n t, \\
\operatorname{var}\left\{y_{n}(x)\right\}=E\left\{y_{n}(x)-n x\right\}^{2}=n x(1-x), \\
\mathbb{B}_{n} f(x)=E\left[f\left\{\frac{y_{n}(x)}{n}\right\}\right],
\end{gathered}
$$

(cf, [7]).

The classical higher-order Bernoulli polynomials $\mathbb{B}_{n}^{(v)}(z)$ are defined by means of the following generating function:

$$
F^{(v)}(z, t)=e^{t x}\left(\frac{t}{e^{t}-1}\right)^{v}=\sum_{n=0}^{\infty} B_{n}^{(v)}(z) \frac{t^{n}}{n !}
$$

The higher-order Bernoulli polynomials play an important role in the finite differences and in (analytic) number theory. So, the coefficients in all the usual central-difference formulae for interpolation, numerical differentiation, and integration and differences in terms of derivatives can be expressed in terms of these polynomials (cf, [2, 11, 12, 24]). These polynomials are related to the many branches of Mathematics. By substituting $v=1$ into the above, we have

$$
F(t)=\frac{t e^{t x}}{e^{t}-1}=\sum_{n=1}^{\infty} B_{n} \frac{t^{n}}{n !}
$$

where $B_{n}$ is usual Bernoulli polynomials (cf, [22]).

The usual Stirling numbers of the second-kind with parameters $(n, k)$ are denoted by $S(n, k)$, that is, the number of partitions of the set $\{1,2, \ldots, n\}$ into $k$ nonempty set. For any $t$, it is well known that the Stirling numbers of the second-kind are defined by means of the generating function (cf, $[3,21,23])$

$$
F_{S}(t, k)=\frac{(-1)^{k}}{k !}\left(1-e^{t}\right)^{k}=\sum_{n=0}^{\infty} S(n, k) \frac{t^{n}}{n !}
$$

These numbers play an important role in many branches of Mathematics, for example, combinatorics, number theory, discrete probability distributions for finding higher-order moments. In [8], Joarder and Mahmood demonstrated the application of the Stirling numbers of the second-kind in calculating moments of some discrete distributions, which are binomial distribution, geometric distribution, and negative binomial distribution. 
The Hermite polynomials are defined by the following generating function.

For $z, t \in \mathbb{C}$,

$$
e^{2 z t-t^{2}}=\sum_{n=0}^{\infty} H_{n}(z) \frac{t^{n}}{n !}
$$

which gives the Cauchy-type integral

$$
H_{n}(z)=\frac{n !}{2 \pi i} \int_{C} e^{2 z t-t^{2}} \frac{d t}{t^{n+1}}
$$

where $C$ is a circle around the origin and the integration is in positive direction (cf, [12]). The Hermite polynomials play a crucial role in certain limits of the classical orthogonal polynomials. These polynomials are related to the higher-order Bernoulli polynomials, Gegenbauer polynomials, Laguerre polynomials, the Tricomi-Carlitz polynomials and Buchholz polynomials, (cf, [12]). These polynomials also play a crucial role not only in Mathematics but also in Physics and in the other sciences. In Section 4 we give relation between the Hermite polynomials and $(q-)$ Bernstein-type polynomials.

\section{Generating Function of the Bernstein-Type Polynomials}

Let $\left\{B_{k, n}(x)\right\}_{0 \leq k \leq n}$ be a sequence of Bernstein polynomials. The aim of this section is to construct generating function of the sequence $\left\{B_{k, n}(x)\right\}_{0 \leq k \leq n}$. It is well known that most of generating functions are obtained from the recurrence formulae. However, we do not use the recurrence formula of the Bernstein polynomials for constructing generating function of them.

We now give the following notation:

$$
[x]=[x: q]= \begin{cases}\frac{1-q^{x}}{1-q}, & q \neq 1 \\ x, & q=1\end{cases}
$$

If $q \in \mathbb{C}$, then we assume that $|q|<1$.

We define

$$
F_{k, q}(t, x)=(-1)^{k} t^{k} \exp ([1-x] t) \times \sum_{m, l=0}\left(\begin{array}{c}
k+l-1 \\
l
\end{array}\right) \frac{q^{l} S(m, k)(x \log q)^{m}}{m !}
$$

where $|q|<1, \exp (x)=e^{x}$ and $S(m, k)$ denotes the second-kind Stirling numbers and

$$
\sum_{m, l=0} f(m) g(l)=\sum_{m=0}^{\infty} f(m) \sum_{l=0}^{\infty} g(l)
$$


By (3.2), we define the following new generating function of polynomial $Y_{n}(k ; x ; q)$ by

$$
F_{k, q}(t, x)=\sum_{n=k}^{\infty} Y_{n}(k ; x ; q) \frac{t^{n}}{n !}
$$

where $t \in \mathbb{C}$.

Observe that if $q \rightarrow 1$ in (3.4), we have

$$
Y_{n}(k ; x ; q) \longrightarrow B_{k, n}(x)
$$

Hence

$$
F_{k}(t, x)=\sum_{n=k}^{\infty} B_{k, n}(x) \frac{t^{n}}{n !}
$$

From (3.4), we obtain the following theorem.

Theorem 3.1. Let $n$ be a positive integer with $k \leq n$. Then one has

$$
\begin{aligned}
Y_{n}(k ; x ; q)= & \left(\begin{array}{l}
n \\
k
\end{array}\right) \frac{(-1)^{k} k !}{(1-q)^{n-k}} \\
& \times \sum_{m, l=0} \sum_{j=0}^{n-k}\left(\begin{array}{c}
k+l-1 \\
l
\end{array}\right)\left(\begin{array}{c}
n-k \\
k
\end{array}\right) \frac{(-1)^{j} q^{l+j(1-x)} S(m, k)(x \log q)^{m}}{m !} .
\end{aligned}
$$

By using (3.2) and (3.4), we obtain

$$
F_{k, q}(t, x)=\frac{([x] t)^{k}}{k !} \exp ([1-x] t)=\sum_{n=k}^{\infty} Y_{n}(k ; x ; q) \frac{t^{n}}{n !}
$$

The generating function $F_{k, q}(t, x)$ depends on integer parameter $k$, real variable $x$, and complex variable $q$ and $t$. Therefore the properties of this function are closely related to these variables and parameter. By using this function, we give many properties of the $(q-)$ Bernstein-type polynomials and the other well-known special numbers and polynomials. By applying Mellin transformation to this function, in Section 5, we construct interpolation function of the $(q-)$ Bernstein-type polynomials.

By the umbral calculus convention in (3.8), then we obtain

$$
\frac{([x] t)^{k}}{k !} \exp ([1-x] t)=\exp (Y(k ; x ; q) t)
$$

By using the above, we obtain all recurrence formulae of $Y_{n}(k ; x ; q)$ as follows:

$$
\frac{([x] t)^{k}}{k !}=\sum_{n=0}^{\infty}(Y(k ; x ; q)-[1-x])^{n} \frac{t^{n}}{n !},
$$


where each occurrence of $Y^{n}(k ; x ; q)$ are given by $Y_{n}(k ; x ; q)$ (symbolically $Y^{n}(k ; x ; q) \rightarrow$ $\left.Y_{n}(k ; x ; q)\right)$.

By (3.9),

$$
[u+v]=[u]+q^{u}[v], \quad[-u]=-q^{u}[u]
$$

we obtain the following corollary.

Corollary 3.2. Let $n$ be a positive integer with $k \leq n$. Then one has

$$
Y_{n+k}(k ; x ; q)=\left(\begin{array}{c}
n+k \\
k
\end{array}\right) \sum_{j=0}^{n}(-1)^{j} q^{j(1-x)}[x]^{j+k}
$$

Remark 3.3. By Corollary 3.2, for all $k$ with $0 \leq k \leq n$, we see that

$$
Y_{n+k}(k ; x ; q)=\left(\begin{array}{c}
n+k \\
k
\end{array}\right) \sum_{j=0}^{n}(-1)^{j} q^{j(1-x)}[x]^{j+k}
$$

The polynomials $Y_{n+k}(k ; x ; q)$ are so-called $q$-Bernstein-type polynomials. It is easily seen that

$$
\lim _{q \rightarrow 1} Y_{n+k}(k ; x ; q)=B_{k, n+k}(x)=\left(\begin{array}{c}
n+k \\
k
\end{array}\right) x^{k}(1-x)^{n}
$$

which give us (2.2).

By using derivative operator

$$
\frac{d}{d x}\left(\lim _{q \rightarrow 1} Y_{n+k}(k ; x ; q)\right)
$$

in (3.2), we obtain

$$
\sum_{n=k}^{\infty} \frac{d}{d x}\left(Y_{n}(k ; x ; 1)\right) \frac{t^{n}}{n !}=\sum_{n=k}^{\infty} n Y_{n-1}(k-1 ; x ; 1) \frac{t^{n}}{n !}-\sum_{n=k}^{\infty} n Y_{n-1}(k ; x ; 1) \frac{t^{n}}{n !}
$$

Consequently, we have

$$
\frac{d}{d x}\left(Y_{n}(k ; x ; 1)\right)=n Y_{n-1}(k-1 ; x ; 1)-n Y_{n-1}(k ; x ; 1),
$$

or

$$
\frac{d}{d x}\left(B_{k, n}(x)\right)=n B_{k-1, n-1}(x)-n B_{k, n-1}(x)
$$


Observe that by using our generating function we give different proof of (2.4). defined by

Let $f$ be a function on $[0,1]$. The $(q-)$ Bernstein-type polynomial of degree $n$ is

$$
\mathbb{Y}_{n} f(x)=\sum_{j=0}^{n} f\left(\frac{[j]}{[n]}\right) Y_{n}(j ; x ; q)
$$

where $0 \leq x \leq 1$. $\mathbb{Y}_{n}$ is called the $(q-)$ Bernstein-type operator and $Y_{n}(j ; x ; q), j=0, \ldots, n$, defined in (3.7), are called the $\left(q^{-}\right)$Bernstein-type (basis) polynomials.

\section{New Identities on Bernstein-Type Polynomials, Hermite Polynomials, and the Stirling Numbers of the Second-Kind}

Theorem 4.1. Let $n$ be a positive integer with $k \leq n$. Then one has

$$
Y_{n}(k ; x ; q)=[x]^{k} \sum_{j=0}^{n}\left(\begin{array}{l}
n \\
j
\end{array}\right) B_{j}^{(k)}([1-x]) S(n-j, k),
$$

where $B_{j}^{(k)}(x)$ and $S(n, k)$ denote the classical higher-order Bernoulli polynomials and the Stirling numbers of the second-kind, respectively.

Proof. By using (2.7), (2.9), and (3.4), we obtain

$$
\sum_{n=k}^{\infty} Y_{n}(k ; x ; q) \frac{t^{n}}{n !}=[x]^{k} \sum_{n=0}^{\infty} S(n, k) \frac{t^{n}}{n !} \sum_{n=0}^{\infty} B_{j}^{(k)}([1-x]) \frac{t^{n}}{n !}
$$

By using Cauchy product in the above, we have

$$
\sum_{n=k}^{\infty} Y(k, n ; x ; q) \frac{t^{n}}{n !}=[x]^{k} \sum_{n=0}^{\infty} \sum_{j=0}^{n} B_{j}^{(k)}([1-x]) S(n-j, k) \frac{t^{n}}{j !(n-j) !} .
$$

From the above, we have

$$
\begin{aligned}
\sum_{n=k}^{\infty} Y_{n}(k ; x ; q) \frac{t^{n}}{n !}= & {[x]^{k} \sum_{n=0}^{k-1} \sum_{j=0}^{n} B_{j}^{(k)}([1-x]) S(n-j, k) \frac{t^{n}}{j !(n-j) !} } \\
& +[x]^{k} \sum_{n=k}^{\infty} \sum_{j=0}^{n} B_{j}^{(k)}([1-x]) S(n-j, k) \frac{t^{n}}{j !(n-j) !}
\end{aligned}
$$

By comparing coefficients of $t^{n}$ in both sides of the above equation, we arrive at the desired result. 
Remark 4.2. In [18-20], Phillips gave many results concerning the $q$-integers, and an account of the properties of $q$-Bernstein polynomials. He gave many applications of these polynomials on approximation theory. In [6], Gould gave a different relation between the Bernstein polynomials, generalized Bernoulli polynomials, and the second-kind Stirling numbers. Oruç and Tuncer [15] gave relation between the $q$-Bernstein polynomials and the second-kind $q$-Stirling numbers. In [13], Nowak studied approximation properties for generalized $q$ Bernstein polynomials and also obtained Stancu operators or Phillips polynomials.

From (4.4), we get the following corollary.

Corollary 4.3. Let $n$ be a positive integer with $k \leq n$. Then one has

$$
[x]^{k} \sum_{n=0}^{k-1} \sum_{j=0}^{n} \frac{B_{j}^{(k)}([1-x]) S(n-j, k)}{j !(n-j) !}=0 .
$$

Theorem 4.4. Let $n$ be a positive integer with $k \leq n$. Then one has

$$
H_{n}(1-y)=\frac{k !}{y^{k}} \sum_{n=0}^{\infty} Y_{n+k}(k ; y ; q) \frac{2^{n}}{(n+k) !} .
$$

Proof. By (2.10), we have

$$
e^{2 z t}=\sum_{n=0}^{\infty} \frac{t^{2 n}}{n !} \sum_{n=0}^{\infty} H_{n}(z) \frac{t^{n}}{n !}
$$

By Cauchy product in the above, we obtain

$$
e^{2 z t}=\sum_{n=0}^{\infty}\left(\sum_{j=0}^{n}\left(\begin{array}{l}
n \\
j
\end{array}\right) H_{j}(z)\right) \frac{t^{2 n-j}}{n !}
$$

By substituting $z=1-y$ into (4.8), we have

$$
\sum_{n=0}^{\infty}\left(\sum_{j=0}^{n}\left(\begin{array}{l}
n \\
j
\end{array}\right) H_{j}(1-y)\right) \frac{t^{2 n-j}}{n !}=\frac{k !}{y^{k}} \sum_{n=0}^{\infty}\left(2^{n} Y_{n+k}(k ; y ; q)\right) \frac{t^{n}}{(n+k) !} .
$$

By comparing coefficients of $t^{n}$ in the both sides of the above equation, we arrive at the desired result.

\section{Interpolation Function of the (q-) Bernstein-Type Polynomials}

The classical Bernoulli numbers interpolate by Riemann' zeta function, which has a profound effect on number theory and complex analysis. Thus, we construct interpolation function of the $(q-)$ Bernstein-type polynomials. 
For $z \in \mathbb{C}$, and $x \neq 1$, by applying the Mellin transformation to (3.2), we get

$$
S_{q}(z, k ; x)=\frac{1}{\Gamma(s)} \int_{0}^{\infty} t^{z-k-1} F_{k, q}(-t, x) d t
$$

By using the above equation, we defined interpolation function of the polynomials $Y_{n}(k ; x ; q)$ as follows.

Definition 5.1. Let $z \in \mathbb{C}$ and $x \neq 1$. We define

$$
S_{q}(z, k ; x)=(1-q)^{z-k} \sum_{m, l=0}\left(\begin{array}{c}
z+l-1 \\
l
\end{array}\right) \frac{q^{l(1-x)} S(m, k)(x \log q)^{m}}{m !} .
$$

By using (5.2), we obtain

$$
S_{q}(z, k ; x)=\frac{(-1)^{k}}{k !}[x]^{k}[1-x]^{-z},
$$

where $z \in \mathbb{C}$ and $x \neq 1$.

By (5.2), we have $S_{q}(z, k ; x) \rightarrow S(z, k ; x)$ as $q \rightarrow 1$. Thus one has

$$
S(z, k ; x)=\frac{(-1)^{k}}{k !} x^{k}(1-x)^{-z}
$$

By substituting $x=1$ into the above, we have

$$
S(z, k ; 1)=\infty
$$

We now evaluate the $m$ th $z$-derivatives of $S(z, k ; x)$ as follows:

$$
\frac{\partial^{m}}{\partial z^{m}} S(z, k ; x)=\log ^{m}\left(\frac{1}{1-x}\right) S(z, k ; x)
$$

where $x \neq 1$.

By substituting $z=-n$ into (5.2), we obtain

$$
S_{q}(-n, k ; x)=\frac{1}{(1-q)^{n+k}} \sum_{m, l=0}\left(\begin{array}{c}
-n+l-1 \\
l
\end{array}\right) \frac{q^{l(1-x)} S(m, k)(x \log q)^{m}}{m !} .
$$

By substituting (3.7) into the above, we arrive at the following theorem, which relates the polynomials $Y_{n+k}(k ; x ; q)$ and the function $S_{q}(z, k ; x)$. 
Theorem 5.2. Let $n$ be a positive integer with $k \leq n$ and $0<x<1$. Then we have

$$
S_{q}(-n, k ; x)=\frac{(-1)^{k} n !}{(n+k) !} Y_{n+k}(k ; x ; q) .
$$

Remark 5.3. Consider the following.

$$
\begin{aligned}
\lim _{q \rightarrow 1} S_{q}(-n, k ; x) & =S(-n, k ; x) \\
& =\frac{(-1)^{k} n !}{(n+k) !} x^{k}(1-x)^{n} \\
& =\frac{(-1)^{k} n !}{(n+k) !} B_{k, n+k}(x) .
\end{aligned}
$$

Therefore, for $0<x<1$, the function

$$
S(z, k ; x)=\frac{(-1)^{k}}{k !} x^{k}(1-x)^{-z}
$$

interpolates the classical Bernstein polynomials of degree $n$ at negative integers.

By substituting $z=-n$ into (5.6), we obtain the following corollary.

Corollary 5.4. Let $n$ be a positive integer with $k \leq n$ and $0<x<1$. Then one has

$$
\frac{\partial^{m}}{\partial z^{m}} S(-n, k ; x)=\frac{(-1)^{k} n !}{(n+k) !} B_{k, n+k}(x) \log ^{m}\left(\frac{1}{1-x}\right) .
$$

\section{Further Remarks and Observation}

The Bernstein polynomials are used for important applications in many branches of Mathematics and the other sciences, for instance, approximation theory, probability theory, statistic theory, number theory, the solution of the differential equations, numerical analysis, constructing Bezier curve, $q$-analysis, operator theory, and applications in computer graphics. Thus we look for the applications of our new functions and the $(q-)$ Bernstein-type polynomials.

Due to Oruç and Tuncer [15], the $q$-Bernstein polynomials share the well-known shape-preserving properties of the classical Bernstein polynomials. When the function $f$ is convex, then

$$
\beta_{n-1}(f, x) \geq \beta_{n}(f, x) \text { for } n>1,0<q \leq 1 \text {, }
$$


where

$$
\begin{gathered}
\beta_{n}(f, x)=\sum_{r=0}^{n} f_{r}\left[\begin{array}{l}
n \\
r
\end{array}\right] x^{r} \prod_{s=0}^{n-r-1}\left(1-q^{s} x\right), \\
{\left[\begin{array}{l}
n \\
r
\end{array}\right]=\frac{[n] \cdots[n-r+1]}{[r] !} .}
\end{gathered}
$$

As a consequence of this one can show that the approximation to convex function by the $q$ Bernstein polynomials is one sided, with $\beta_{n} f \geq f$ for all $n$. $\beta_{n} f$ behaves in very nice way when one varies the parameter $q$. In [2], the authors gave some applications on the approximation theory related to Bernoulli and Euler polynomials.

We conclude this section by the following questions.

(1) How can one demonstrate approximation by $(q-)$ Bernstein-type polynomials $Y_{n+k}(k ; x ; q)$ ?

(2) Is it possible to define uniform expansions of the $(q-)$ Bernstein-type polynomials $Y_{n+k}(k ; x ; q)$ ?

(3) Is it possible to give applications of the $(q-)$ Bernstein-type polynomials in calculating moments of some distributions in Statistics $Y_{n+k}(k ; x ; q)$ ?

(4) How can one give relations between the $(q-)$ Bernstein-type polynomials $Y_{n+k}(k ; x ; q)$ and the Milnor algebras.

\section{Acknowledgments}

The first author is supported by the research fund of Akdeniz University. The authors would like to thank the referee for his/her comments. The authors also would like to thank Professor George M. Phillips for his comments and his some references.

\section{References}

[1] S. N. Bernstein, "Démonstration du théorème de Weierstrass fondée sur la calcul des probabilités," Communications of the Mathematical Society of Charkow. Séries 2, vol. 13, pp. 1-2, 1912-1913.

[2] M. Acikgoz and Y. Simsek, "On multiple interpolation functions of the Nörlund-type q-Euler polynomials," Abstract and Applied Analysis, vol. 2009, Article ID 382574, 14 pages, 2009.

[3] N. P. Cakić and G. V. Milovanović, "On generalized Stirling numbers and polynomials," Mathematica Balkanica, vol. 18, no. 3-4, pp. 241-248, 2004.

[4] S. Berg, "Some properties and applications of a ratio of Stirling numbers of the second kind," Scandinavian Journal of Statistics, vol. 2, no. 2, pp. 91-94, 1975.

[5] T. N. T. Goodman, H. Oruç, and G. M. Phillips, "Convexity and generalized Bernstein polynomials," Proceedings of the Edinburgh Mathematical Society, vol. 42, no. 1, pp. 179-190, 1999.

[6] H. W. Gould, "A theorem concerning the Bernstein polynomials," Mathematics Magazine, vol. 31, no. 5, pp. 259-264, 1958.

[7] Z. Guan, "Iterated Bernstein polynomial approximations," http://arxiv.org/abs/0909.0684.

[8] A. H. Joarder and M. Mahmood, "An inductive derivation of Stirling numbers of the second kind and their applications in statistics," Journal of Applied Mathematics E Decision Sciences, vol. 1, no. 2, pp. 151-157, 1997.

[9] K. I. Joy, “Bernstein polynomials, On-Line Geometric Modeling Notes," http://en.wikipedia.org/ wiki/Bernstein_polynomial. 
[10] E. Kowalski, “Bernstein polynomials and Brownian motion," American Mathematical Monthly, vol. 113, no. 10, pp. 865-886, 2006.

[11] N. E. Nörlund, Vorlesungen über Differenzenrechung, Springer, Berlin, Germany, 1924.

[12] J. L. López and N. M. Temme, "Hermite polynomials in asymptotic representations of generalized Bernoulli, Euler, Bessel, and Buchholz polynomials," Journal of Mathematical Analysis and Applications, vol. 239, no. 2, pp. 457-477, 1999.

[13] G. Nowak, "Approximation properties for generalized q-Bernstein polynomials," Journal of Mathematical Analysis and Applications, vol. 350, no. 1, pp. 50-55, 2009.

[14] H. Oruç and G. M. Phillips, "A generalization of the Bernstein polynomials," Proceedings of the Edinburgh Mathematical Society, vol. 42, no. 2, pp. 403-413, 1999.

[15] H. Oruç and N. Tuncer, "On the convergence and iterates of $q$-Bernstein polynomials," Journal of Approximation Theory, vol. 117, no. 2, pp. 301-313, 2002.

[16] S. Ostrovska, "The approximation by $q$-Bernstein polynomials in the case $q \downarrow 1$," Archiv der Mathematik, vol. 86, no. 3, pp. 282-288, 2006.

[17] S. Ostrovska, "On the q-Bernstein polynomials," Advanced Studies in Contemporary Mathematics, vol. 11, no. 2, pp. 193-204, 2005.

[18] G. M. Phillips, "Bernstein polynomials based on the $q$-integers, the heritage of P. L. Chebyshev: a Festschrift in honor of the 70th birthday of T. J. Rivlin," Annals of Numerical Mathematics, vol. 4, no. 1-4, pp. 511-518, 1997.

[19] G. M. Phillips, Interpolation and Approximation by Polynomials, vol. 14 of CMS Books in Mathematics/Ouvrages de Mathématiques de la SMC, Springer, New York, NY, USA, 2003.

[20] G. M. Phillips, "A survey of results on the $q$-Bernstein polynomials," IMA Journal of Numerical Analysis, vol. 30, no. 1, pp. 277-288, 2010.

[21] Á. Pintér, "On a Diophantine problem concerning Stirling numbers," Acta Mathematica Hungarica, vol. 65, no. 4, pp. 361-364, 1994.

[22] Y. Simsek, "Twisted $(h, q)$-Bernoulli numbers and polynomials related to twisted $(h, q)$-zeta function and L-function," Journal of Mathematical Analysis and Applications, vol. 324, no. 2, pp. 790-804, 2006.

[23] Y. Simsek, "On q-deformed Stirling numbers," http:/ / arxiv.org/abs/0711.0481.

[24] Y. Simsek, V. Kurt, and D. Kim, "New approach to the complete sum of products of the twisted $(h, q)$-Bernoulli numbers and polynomials," Journal of Nonlinear Mathematical Physics, vol. 14, no. 1 pp. 44-56, 2007.

[25] Z. Wu, "The saturation of convergence on the interval $[0,1]$ for the $q$-Bernstein polynomials in the case $q>1$," Journal of Mathematical Analysis and Applications, vol. 357, no. 1, pp. 137-141, 2009. 


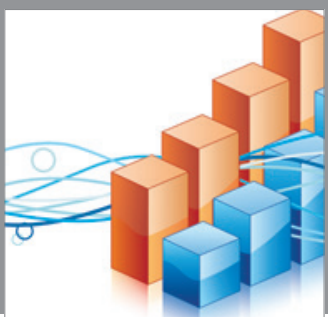

Advances in

Operations Research

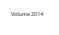

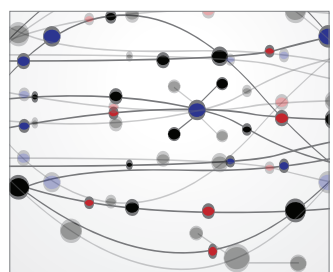

\section{The Scientific} World Journal
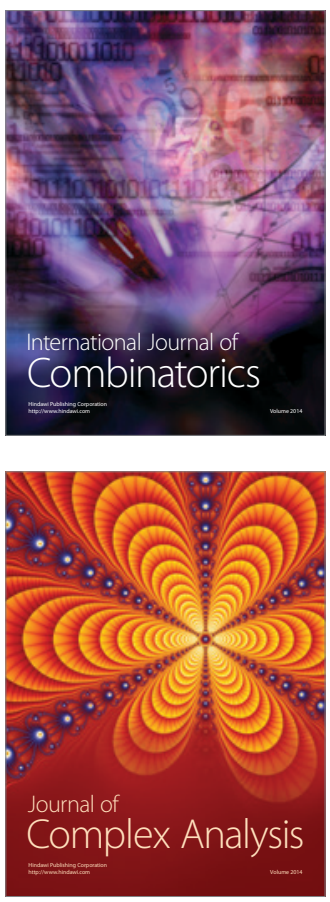

International Journal of

Mathematics and

Mathematical

Sciences
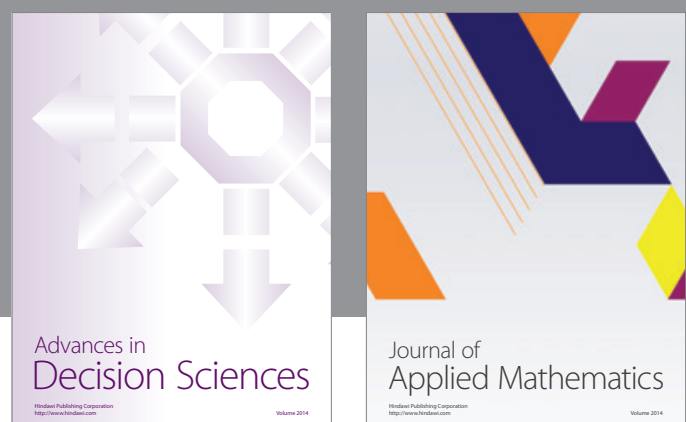

Journal of

Applied Mathematics
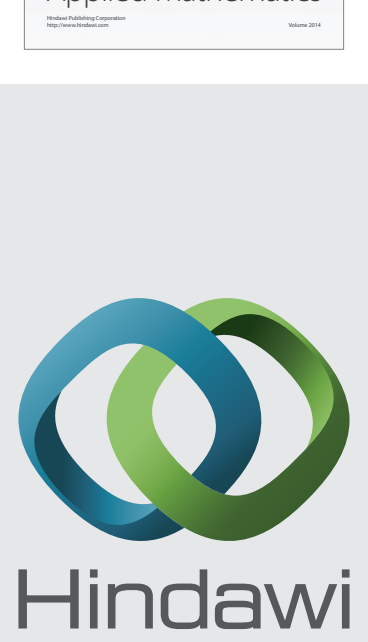

Submit your manuscripts at http://www.hindawi.com
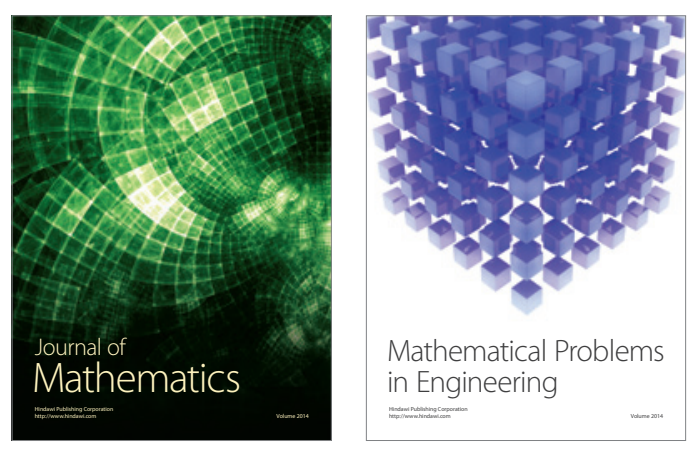

Mathematical Problems in Engineering
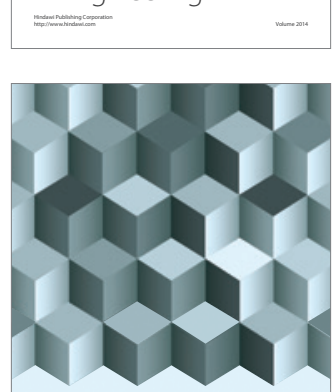

Journal of

Function Spaces
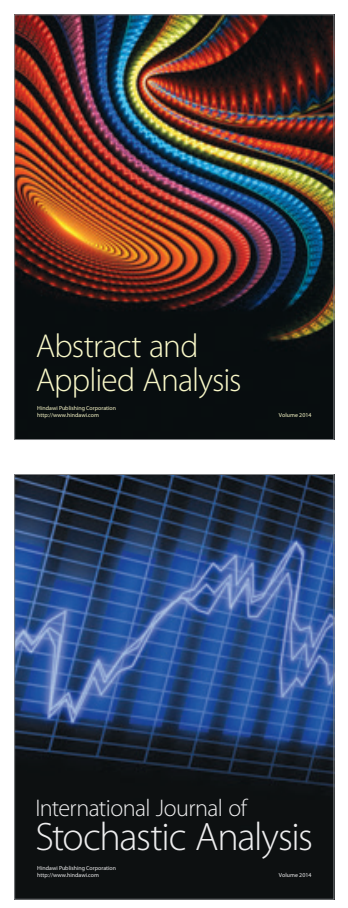

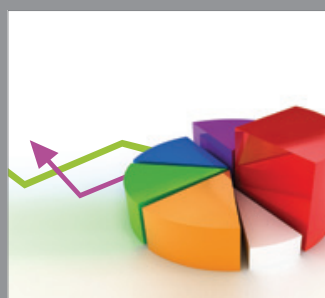

ournal of

Probability and Statistics

Promensencen
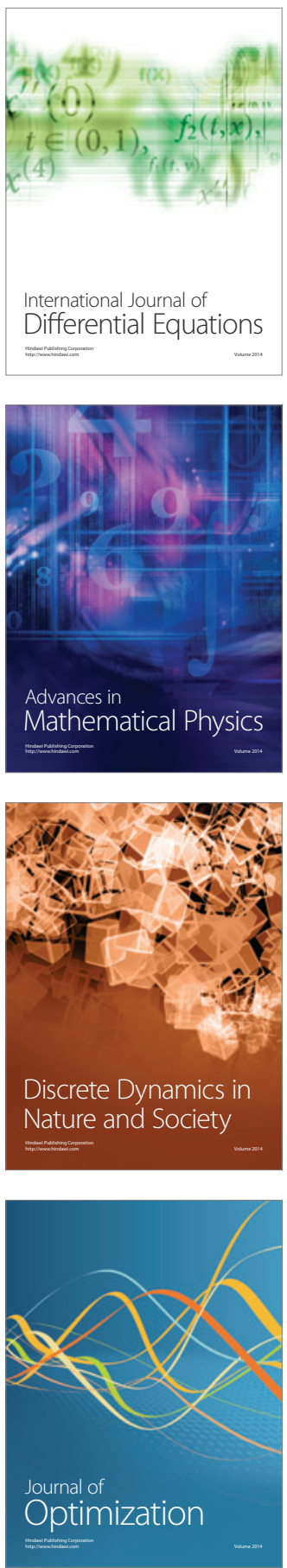